\title{
Temporal and micro-spatial heterogeneity in the distribution of Anopheles vectors of malaria along the Kenyan coast
}

\author{
Martin Walker ${ }^{1 \dagger}$, Peter Winskill ${ }^{1,2 \dagger}$, María-Gloria Basáñez ${ }^{1 *}$, Joseph M Mwangangi ${ }^{3}$, Charles Mbogo ${ }^{3}$,
} John C Beier ${ }^{4}$ and Janet T Midega ${ }^{3,5}$

\begin{abstract}
Background: The distribution of anopheline mosquitoes is determined by temporally dynamic environmental and human-associated variables, operating over a range of spatial scales. Macro-spatial short-term trends are driven predominantly by prior (lagged) seasonal changes in climate, which regulate the abundance of suitable aquatic larval habitats. Micro-spatial distribution is determined by the location of these habitats, proximity and abundance of available human bloodmeals and prevailing micro-climatic conditions. The challenge of analysing -in a single coherent statistical framework - the lagged and distributed effect of seasonal climate changes simultaneously with the effects of an underlying hierarchy of spatial factors has hitherto not been addressed.
\end{abstract}

Methods: Data on Anopheles gambiae sensu stricto and A. funestus collected from households in Kilifi district, Kenya, were analysed using polynomial distributed lag generalized linear mixed models (PDL GLMMs).

Results: Anopheline density was positively and significantly associated with amount of rainfall between 4 to 47 days, negatively and significantly associated with maximum daily temperature between 5 and 35 days, and positively and significantly associated with maximum daily temperature between 29 and 48 days in the past (depending on Anopheles species). Multiple-occupancy households harboured greater mosquito numbers than single-occupancy households. A significant degree of mosquito clustering within households was identified.

Conclusions: The PDL GLMMs developed here represent a generalizable framework for analysing hierarchically-structured data in combination with explanatory variables which elicit lagged effects. The framework is a valuable tool for facilitating detailed understanding of determinants of the spatio-temporal distribution of Anopheles. Such understanding facilitates delivery of targeted, cost-effective and, in certain circumstances, preventative antivectorial interventions against malaria.

Keywords: Anopheles gambiae, Anopheles funestus, Polynomial distributed lag generalized linear mixed models, Rainfall, Temperature, Household occupancy, Larval habitats, Mosquito density, Malaria, Kilifi, Kenya

\section{Background}

The density of Anopheles mosquito vectors relative to human hosts (the vector-to-host ratio) is a critical component of the intensity of malaria transmission [1]. Spatial and temporal heterogeneity in the density and distribution of anopheline mosquitoes is determined principally by the availability of aquatic habitats suitable for the maturation

\footnotetext{
*Correspondence: m.basanez@imperial.ac.uk

${ }^{\dagger}$ Equal contributors

'Department of Infectious Disease Epidemiology, School of Public Health, Faculty of Medicine (St Mary's campus), Imperial College London, Norfolk Place, London W2 1PG, UK

Full list of author information is available at the end of the article
}

and development of their larvae [2,3]. Climate is a major determinant of macro-spatial (district, regional or country) and temporal heterogeneity in the distribution of such habitats and consequently of heterogeneity in the distribution of mosquito vectors [4-8]. In turn, climatic variation drives heterogeneity in the intensity of malaria transmission within and among human populations, both in the short(seasonal) [9] and long- (climate change) terms [10]. The fine, micro-spatial distribution of vectors within communities depends on the location of aquatic habitats $[11,12]$, the proximity of human bloodmeals [13,14] (Anopheles gambiae sensu stricto and $A$. funestus mosquitoes are strongly

\section{Biomed Central}


anthropophagic [15]) and micro-climatic conditions, particularly the direction of the prevailing wind [16] that transports olfactory cues from humans to female mosquitoes $[17,18]$.

There is great interest in identifying factors underlying the macro- and micro-spatial and temporal distribution of anopheline vectors either mechanistically using mathematical models (e.g. [19-21]), or phenomenologically using statistical models (e.g. [22-24]). Studies are often motivated by projecting the effects of climate change on the distribution of anophelines [25-27] and endemic malaria [28]; improving targeted control on macro- $[29,30]$ and microspatial $[16,31]$ scales, or developing models with capacity to offer reliable predictions of impending malaria epidemics [32]. The latter 'early warning' prediction models are underpinned by the lagged relationship between meteorological variables, vector density and malaria incidence [33]. For example, depending on temperature, it takes between 6 and 47 days for mosquito larvae to develop into adults; between 4 and 111 days for the completion of sporogony within a mosquito following ingestion of an infected bloodmeal (infected mosquitoes are not infectious before completion of sporogony), and a further 10- to 16-day incubation period before an infected human develops symptoms of malaria (see Table 1 in [34]). Thus, increased anopheline densities [35,36], and cases of malaria [34], are associated with prior increases in rainfall [37].

Despite an extensive literature, there remains a lack of general consensus on the relative importance and predictive capacity of different meteorological factors [35]. This is partly due to the biological and ecological complexities that underpin the associations. For example, the association between increased rainfall and increased vector abundance would appear ostensibly simple, being mediated by more numerous and larger aquatic habitats. However, the persistence of such habitats following rain also depends on rates of evaporation, which are themselves driven by a myriad of factors, including temperature, atmospheric pressure, wind-speed, humidity and the surface area of the specific habitat [19]. Furthermore, temperaturedependent rates of larval development [38-40] ensure that the lag between increased rainfall and increased abundance of mosquitoes will vary with temperature; at an average temperature of $16^{\circ} \mathrm{C}$ larvae become adults in an average of
47 days, while at $30^{\circ} \mathrm{C}$ it takes an average of 10 days (see Table 1 in [34] and Figure 1 in [41]). Moreover, when inference is made using data on malaria incidence, temperaturedependent rates of sporogony and the incubation period in the human, add an additional and uncertain lag time.

Methodological issues related to the analysis of these complex associations are also likely to contribute to somewhat conflicting conclusions on the relative importance of different meteorological variables. The most common analytical approach has been to calculate pairwise correlations between malaria incidence and variables such as daily rainfall (e.g. [42]), temperature (e.g. [43]) and humidity (e.g. [44]) at different numbers of days in the past (lags). This univariate approach is not conducive to multivariate adaptation, making it prone to confounding by inevitably (highly) correlated meteorological variables. Furthermore, the method does not quantify the overall statistical significance nor the explanatory power of variables considered at different lags.

Polynomial distributed lag (PDL) models [45] provide a solution to these analytical difficulties, permitting the association of multiple explanatory (meteorological) variables over a continuum of lags to be estimated as part of a single, coherent model structure. Such models have been used previously to estimate the association of rainfall, and daily temperature with weekly incidence of malaria in Ethiopia distributed over a 10 -week lag period [34]. The PDL framework can be applied to explanatory variables in a generalized linear model (GLM) [46]. The resulting PDL GLM is adaptable to hierarchical data structures, becoming a polynomial distributed lag generalized linear mixed model (PDL GLMM, Table 2) [47,48]. Such frameworks permit modelling of explanatory variables acting on a variety of scales; from climatic (meteorological) data acting at the macro-spatial scale, to temporally-dependent factors acting below the level of the (longitudinally sampled) units of observation. This potential of the PDL GLMM framework has not been applied to the analysis of data on vector abundance, and consequently little is known about the relative importance and predictive capacity of variables acting on multiple hierarchical scales.

In this paper, mixed (containing random effects) PDL models (Table 2) are used to analyse longitudinal data on the abundance of Anopheles gambiae and A. funestus,

Table 1 Descriptive statistics of the three sampled study villages in Kilifi district, Kenya

\begin{tabular}{|c|c|c|c|c|c|c|}
\hline \multirow[t]{2}{*}{ Village } & \multirow[t]{2}{*}{$\begin{array}{l}\text { Number of } \\
\text { samples/households }\end{array}$} & \multirow[t]{2}{*}{$\begin{array}{l}\text { Mean }{ }^{\mathrm{a}} / \text { range visits } \\
\text { per household }\end{array}$} & \multirow{2}{*}{$\begin{array}{l}\text { Mean }{ }^{a} / \text { range distance } \\
\text { from nearest mosquito } \\
\text { larval habitat }(\mathrm{m})\end{array}$} & \multirow[t]{2}{*}{$\begin{array}{l}\text { Mean }{ }^{\mathrm{a}} / \text { range } \\
\text { household occupancy }\end{array}$} & \multicolumn{2}{|c|}{$\begin{array}{l}\text { Total/mean }{ }^{\mathrm{a}} / \text { variance }^{\mathrm{a}} \text { number } \\
\text { of female Anopheles collected }\end{array}$} \\
\hline & & & & & A. gambiae & A. funestus \\
\hline Jaribuni & $786 / 12$ & $66 / 4-95$ & $350 / 52-620$ & $3.1 / 1-9$ & $2,422 / 3.1 / 58$ & $5,199 / 6.6 / 380$ \\
\hline Majajani & $948 / 23$ & $41 / 1-86$ & $220 / 52-630$ & $2.9 / 1-9$ & $1,911 / 2.0 / 52$ & $495 / 0.52 / 11$ \\
\hline Mtepeni & $926 / 19$ & $49 / 1-96$ & $170 / 40-540$ & $2.8 / 1-12$ & $1,187 / 1.3 / 14$ & $411 / 0.44 / 2.5$ \\
\hline
\end{tabular}

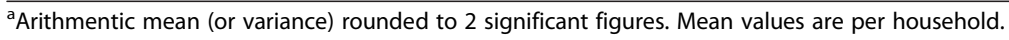



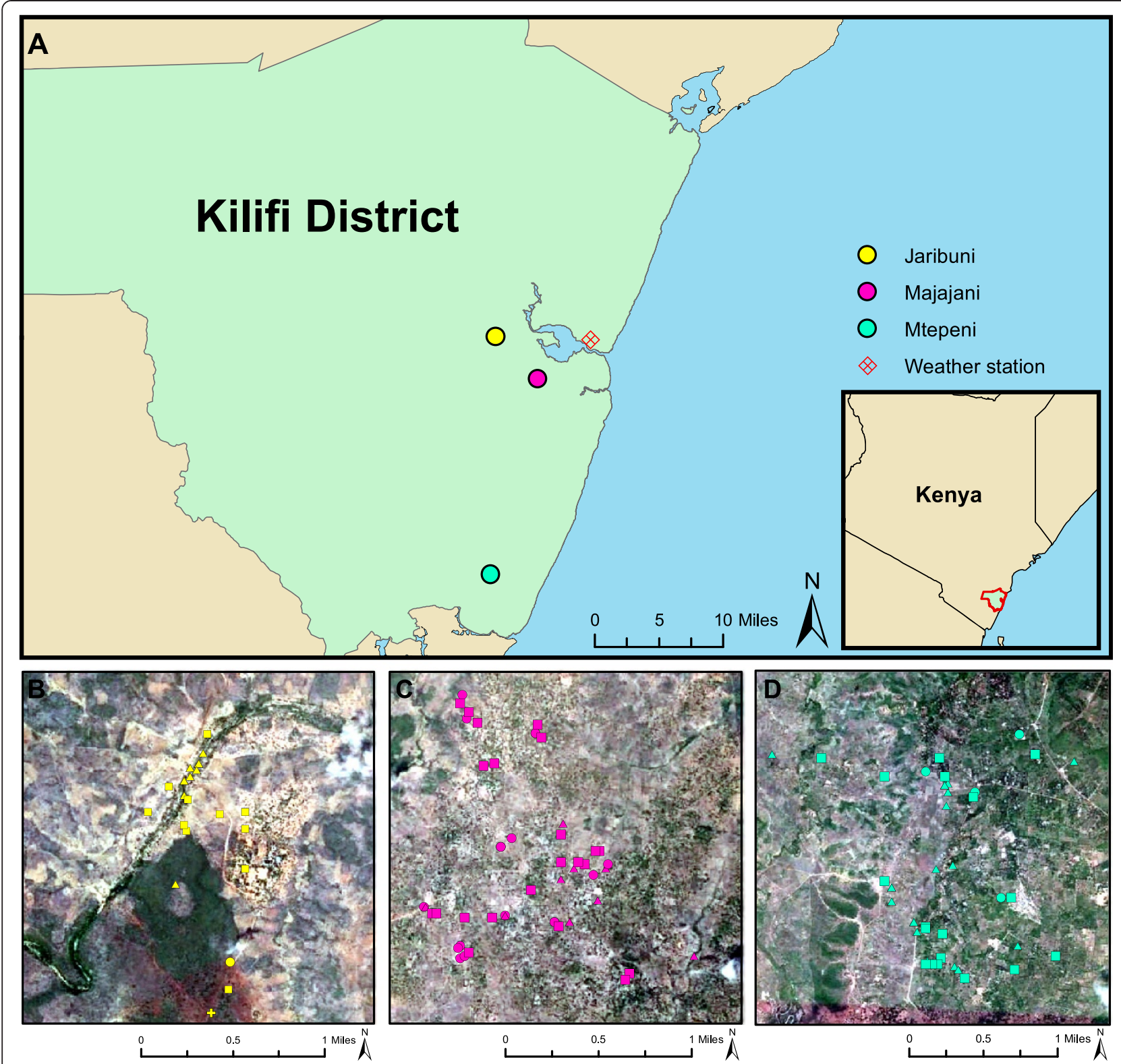

\section{Breeding sites}

\section{Permanent $\square$ Houses \\ Semi-permanent \\ फ Temporary}

Figure 1 Locations of the studied communities, households and anopheline larval habitats. Panel A depicts the locations of the studied communities of Jaribuni, Majajani and Mtepeni within Kilifi district, which is located in south-east Kenya (inset). The location of the national weather station (within the town of Kilifi) from which the meteorological data were collected is also indicted (grid marker). Panels B, C and D are, respectively, satellite images of Jaribuni (yellow markers), Majajani (pink markers) and Mtepeni (green markers) overlaid with the locations of the sampled households (square markers) and anopheline larval habitats. Larval habitats are classified as permanent (e.g. riverbanks, large ponds and swamps; circles); semi-permanent (e.g. streams, pools, and small ponds; triangles), or temporary (e.g. small streams, and small pools created by tire tracks and damaged infrastructure; crosses).

collected between 2000 and 2002 from households located in Kilifi district, adjoining the Kenyan coast, an area endemic for falciparum malaria. Research on anopheline mosquitoes in Kilifi, ongoing since the early 1990s [23,49-54], has identified the importance of environmental heterogeneity in affecting the abundance and productivity of mosquito larval habitats [55], the distribution of adult mosquitoes, and the incidence of malaria 
Table 2 Polynomial distributed lag generalized linear mixed models (PDL GLMMs)

\begin{abstract}
Generalized linear mixed models (GLMMs)
Generalized linear models (GLMs) extend general linear regression models to the analysis of data with non-normally distributed error structures arising from the exponential family of probability distributions. Discrete (count) distributions within this family include the binomial, Poisson, and negative binomial distribution with known overdispersion parameter $k$ Generalized linear mixed models (GLMMs) are GLMs that include both fixed and random effects. Fixed effects are represented by a measured explanatory variable (covariate) and are quantified by regression coefficients. By contrast, random effects embody the unmeasured or unmeasurable characteristics of a unit of observation which induce correlation (clustering) among data collected from the same unit; e.g. numbers of mosquitoes collected from the same household. Random effects are quantified in terms of variability (variance) among data collected from distinct units of observation.
\end{abstract}

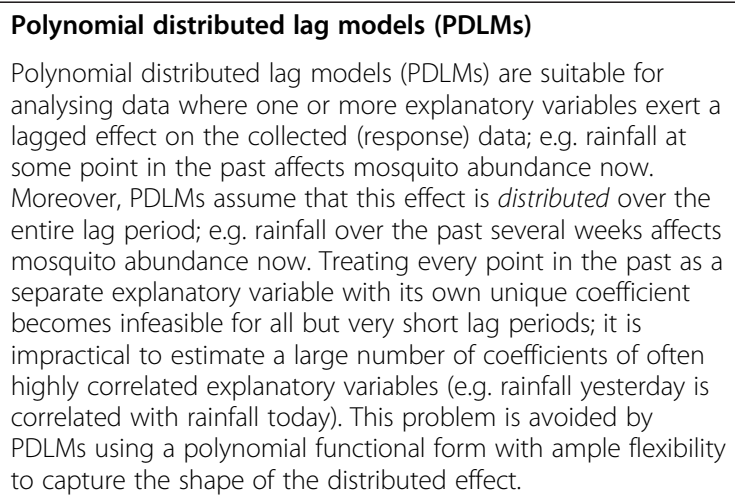

Polynomial distributed lag models (PDLMs) are suitable for analysing data where one or more explanatory variables exert a lagged effect on the collected (response) data; e.g. rainfall at some point in the past affects mosquito abundance now. Moreover, PDLMs assume that this effect is distributed over the entire lag period; e.g. rainfall over the past several weeks affects mosquito abundance now. Treating every point in the past as a separate explanatory variable with its own unique coefficient becomes infeasible for all but very short lag periods; it is impractical to estimate a large number of coefficients of often highly correlated explanatory variables (e.g. rainfall yesterday is correlated with rainfall today). This problem is avoided by PDLMs using a polynomial functional form with ample flexibility to capture the shape of the distributed effect.

due to Plasmodium falciparum $[23,56]$, the latter having declined in recent years $[57,58]$.

The PDL GLMM models presented here incorporate the (lagged) meteorological covariates of daily rainfall and maximum temperature, and the household-level covariates of distance from the nearest aquatic larval habitat and occupancy (number of people in the household) with the aim of: a) exemplifying the flexibility and power of the PDL GLMM framework to analyse longitudinal, hierarchical and overdispersed count data; b) identifying factors underlying the observed spatial and temporal patterns as a source of baseline information prior to the recent decline in malaria transmission and vector density in Kilifi [59], and c) providing information which may influence the design and application of targeted, costeffective and preventative anti-vectorial interventions for malaria control.

\section{Methods}

\section{Study area, household sampling, and ethical considerations}

The study was conducted in the villages of Jaribuni $\left(39^{\circ}\right.$ 44'E $3^{\circ} 37^{\prime} \mathrm{S}$ ) (site 1), Majajani (3974'E 340'S) (site 2), and Mtepeni $\left(39^{\circ} 45^{\prime} \mathrm{E} 3^{\circ} 53^{\prime} \mathrm{S}\right.$ ) (site 3) in Kilifi District, along the Kenyan coast over the period from May 2000 through April 2002 (Figure 1A). Random samples of approximately $30 \%$ of all households in each community were selected for potential inclusion in the study. Written informed consent was sought from the heads of households to permit mosquito collection after the study was explained in their local language. Households that did not consent did not participate further. The initial study design was to perform mosquito catches in each participating household fortnightly throughout the two-year study period. However, due to householders sometimes being absent and, more commonly, requesting additional catches, the mean number of catches performed per household varied between 41 and 66 (Table 1). Most households were visited and sampled on multiple occasions over the study, but not more than once per week. The maximum number of visits to a household was 96, the minimum was 1 (Table 1 ). The study was reviewed and approved by the Institutional Review Board of the Kenya Medical Research Institute (KEMRI), Nairobi, Kenya.

\section{Entomological data}

Anopheles mosquitoes were sampled using the pyrethrum spray catch (PSC) method [60]. Mosquitoes from each household were held separately in labelled paper cups, stored in a cool box, and transported to the laboratory. In the laboratory, all mosquitoes were immediately transferred to a $-20^{\circ} \mathrm{C}$ freezer for approximately $10 \mathrm{mi}$ nutes. Each mosquito sample was allocated a unique identification number and separated by morphospecies into $A$. gambiae and $A$. funestus using the morphological criteria of Gillies and Coetzee [61]. The legs and wings of each Anopheles female were then detached from the rest of the body and stored dry on silica gel in labelled vials before being processed for molecular differentiation of the $A$. gambiae and $A$. funestus sibling species using the methods of Scott et al. [62] and Koekemoer et al. [63]. Only A. gambiae sensu stricto (s.s.) (henceforth referred to simply as A. gambiae) was identified among the A. gambiae s.l. samples (i.e., these contained no $A$. arabiensis or $A$. merus, the other members of the gambiae complex that have been reported along the Kenyan coast [23]). Likewise, $A$. funestus was found to comprise A. funestus s.s. and is henceforth referred to as $A$. funestus.

\section{Household covariates}

All households participating in mosquito sampling were mapped using a hand held global positioning system (GPS) device (Garmin International Inc., Olathe, KS USA). Larval habitats within a $1 \mathrm{~km}$ distance from the study households were also mapped (Figures 1B, 1C, 1D). Samples of mosquito larvae were collected from each habitat 
and were morphologically identified as anophelines (as opposed to other culicines). Larvae were not disaggregated by Anopheles species (complexes). Distance from the larval habitats to the nearest household was calculated using $\mathrm{R}$ [64]. In addition, the number of individuals sleeping in the household the night before mosquito sampling was recorded on field data forms during mosquito sampling. Note that the number of individuals sleeping in a household changed over time and so was included at the observationlevel (rather than the household-level) in the statistical model.

\section{Meteorological data}

Daily meteorological data on the rainfall and minimum and maximum temperatures were obtained from the national weather station located at the Kilifi Institute of Agriculture $\left(3^{\circ} 37^{\prime} 15^{\prime \prime} \mathrm{S} 39^{\circ} 50^{\prime} 45^{\prime \prime} \mathrm{E}\right)$ within the town of Kilifi (Figure 1A). The weather station lies 10.8, 11.5 and $34.1 \mathrm{~km}$ to the north east of Jaribuni, Majajani and Mtepeni respectively. This weather station serves the whole of Kilifi district and represents the most accurate source of data available on the climate in the three study communities. For the fewer than $1 \%$ of days that data were missing, values were imputed by linearly interpolating to the midpoint between the preceding and following days for which data were recorded. There were no instances of missing data on more than one consecutive day. It was assumed that the meteorological data represent accurately the climatic conditions in the surrounding study sites.

\section{Statistical framework}

Data on the number of $A$. gambiae or A. funestus were modelled separately using a generalized linear mixed model (GLMM). Let $Y_{i j}$ denote observation $j$ (number of mosquitoes) collected from household $i$. It was assumed that $Y_{i j}$ was Poisson distributed with mean and variance, $\mu_{i j}$, given by the following log-linear mixed effects model,

$$
\begin{aligned}
& \ln \mu_{i j}=\boldsymbol{\alpha} \mathbf{x}_{i j}+\boldsymbol{\beta} \mathbf{z}_{i}+\varepsilon_{i}+e_{i j}, \\
& \varepsilon_{i} \sim N\left(0, \delta_{\mathrm{H}}\right), \\
& e_{i j} \sim N\left(0, \delta_{\mathrm{O}}\right) .
\end{aligned}
$$

Here $\mathbf{x}_{i j}$ and $\mathbf{z}_{i}$ are observation-level and householdlevel vectors of covariates respectively. The covariate vector $\mathbf{x}_{i j}$ comprises the number of people sleeping in the household the night before day $t_{i j}$ after the start of the study, and the daily rainfall and temperature lagged from $p_{\min }$ to $p_{\max }$ days into the past. For example, if $p_{\text {min }}=5$ and $p_{\max }=10, \mathbf{x}_{i j}$ would include the daily rainfall and temperatures 5-10 days prior to day $t_{i j}$. Day $t_{i j}$ is also included as a continuous covariate in $\mathbf{x}_{i j}$. The inclusion of $t_{i j}$ accounts for any systematic (non-seasonal) linear changes in the density of the mosquito population over the study period. Furthermore, this relationship was permitted to vary among sites by including an appropriate interaction term. The household-level covariate vector, $\mathbf{z}_{i}$, comprises the distance of the household from the nearest mosquito larval habitat, and the location (site) of the household (Jaribuni, Majajani or Mtepeni). In the loglinear model the coefficients measure the multiplicative effect on the mean number of mosquitoes per household of the covariate in question.

The error term $\varepsilon_{i}$ in Eqn. (1) is a household-level, normally distributed random effects term that accounts for the possibility that mosquitoes cluster within households. On the natural logarithmic scale, the magnitude of this clustering is quantified by the variance $\delta_{\mathrm{H}}^{2}$. Converting to the scale of counts (as opposed to log counts), the clustering is quantified by $\sigma_{\mathrm{H}}^{2}=\exp \left(2 \delta_{\mathrm{H}}^{2}\right)-\exp \left(\delta_{\mathrm{H}}^{2}\right)$. Household clustering causes extra-Poisson variation (overdispersion) in the numbers of mosquitoes per household. It was particularly important to account for household clustering in this analysis due to the potential for heavily infested households to have been sampled somewhat disproportionately (some householders received by request additional unplanned mosquito catches, see Methods, Study area, household sampling, and ethical considerations). The residual overdispersion that is not due to household clustering is accounted for by the random effects term $e_{i j}$ in Eqn. (1); this parameter is normally distributed, independent of $\varepsilon_{i}$ and specific to each observation. That is, it is an observation-level random effect. On the natural logarithmic scale, the residual overdispersion is quantified by $\delta_{\mathrm{O}}^{2}$. On the original scale of the counts, it is quantified by $\sigma_{\mathrm{O}}^{2}=\exp \left(2 \delta_{\mathrm{O}}^{2}\right)-\exp \left(\delta_{\mathrm{O}}^{2}\right)$. Modelling residual (within household) overdispersion using an observation-level random effect $[65,66]$ has two principal advantages over alternative methods such as negative binomial or quasi-Poisson regression. First, the model is maintained as a GLMM, permitting likelihood-based fitting and coefficient estimation within a well-developed framework $[47,48]$. Second, overdispersion is modelled in a hierarchical manner (observations nested within households) permitting direct comparison of the relative contribution of random variation at observation and household levels (viz. comparison of $\sigma_{\mathrm{H}}^{2}$ and $\sigma_{\mathrm{O}}^{2}$ ).

\section{Polynomial distributed lag model}

In the model described by Eqn. (1), the number of coefficients to estimate for a particular meteorological covariate is equal to the number of lags considered. For example, if daily rainfall and temperature were lagged from 5 to 15 days into the past $\left(p_{\min }=5, p_{\max }=15\right)$, it would be necessary to estimate 10 coefficients for each meteorological covariate. Coefficients estimated using this so-called unconstrained lag structure are unstable because of collinearity among different lags of the same variable [45]. A 
solution to this is to constrain the coefficients by forcing them to take the shape of a function of the lag. The Almon lag model [67] uses an $n^{\text {th }}$ degree polynomial as this functional form,

$$
\beta_{l}=\sum_{k=0}^{k=n} \theta_{k} l^{k}
$$

Here $\beta_{l}$ represents the coefficient of a variable at lag $l$. That is, $\beta_{l}$ is a coefficient of either rainfall, or maximum temperature $l$ days in the past. Parameter $\theta_{k}$ is the $k^{\text {th }}$ coefficient of the $n^{\text {th }}$ degree polynomial. Thus, the number of estimated coefficients (the $\theta_{k} \mathrm{~s}$ ) associated with each lagged meteorological covariate is reduced from the number of lag days to the order of the polynomial, $n$, giving rise to the PDL.

\section{Model fitting}

The model was fitted to the data using GLMM techniques $[47,48]$ implemented using the lme4 package for $\mathrm{R}$ [64]. Following Teklehaimanot et al. [34], the polynomial describing the relationship between the number of lag days and the coefficients of rainfall and temperature was set to order $4(n=4)$. Preliminary analyses indicated that this order polynomial gave ample flexibility to capture variously shaped relationships. The range of lags considered was motivated by mosquito biology. The time from oviposition of eggs in an aquatic habitat to the development of an adult mosquito depends on temperature, varying between about 7 days ( 1 week) at $40^{\circ} \mathrm{C}$ to 47 days (approximately 7 weeks) at $16^{\circ} \mathrm{C}[34,68,69]$.

\section{Results}

\section{Descriptive statistics}

Table 1 gives descriptive statistics of the three study sites; the villages of Jaribuni, Majajani and Mtepeni in Kilifi district, Kenya (Figure 1). Majajani was the most sampled village; 948 mosquito catches were performed in 23 households. The least sampled village was Jaribuni, with 786 catches undertaken in 12 households. The density of Anopheles mosquitoes was highest in Jaribuni; the mean number of $A$. gambiae and A. funestus per household was 3.1 and 6.6 respectively. Overall, Table 1 indicates that there was considerable heterogeneity in the mean Anopheles densities among the three sites. In particular, the mean density of $A$. funestus was thirteen-fold and fifteen-fold larger in Jaribuni compared with Majajani and Mtepeni respectively. The distances of households from the nearest mosquito larval habitats were also heterogeneous among sites. For instance, the average distance of a household from a larval habitat in Mtepeni was approximately half that of the corresponding mean distance in Jaribuni. The average number of people sleeping in a household the night prior to mosquito sampling was broadly similar among sites (Table 1).

\section{Fitted model}

Figure 2 depicts the observed and model-predicted mean Anopheles densities per household in the three study sites. In general, the model fits the observed A. gambiae data well; the model-predicted mean falls within the $95 \%$ confidence intervals of the majority of the observed data across all sites. Similarly, the model also fits well to the observed A. funestus data from Majajani and Mtepeni. The least good fit is to the data on the household densities of $A$. funestus from Jaribuni, where the mean density was particularly high compared with Majajani and Mtepeni (Table 1).

\section{Meteorological covariates}

Rainfall and maximum temperature from 7 weeks before the start of the study period, in April 2000, to its end in April 2002 are depicted in Figure 3. This timeframe is in accordance with the 7-week range of lags considered for these meteorological covariates in the statistical model. The data indicate two pronounced rainy seasons, peaking around May or June. Temperatures tend to be highest between, approximately, November and May, and lowest between, approximately, June and October. It is also clear from Figure 3 that minimum and maximum daily temperature are strongly positively correlated (Pearson's correlation coefficient $=0.77$ ). This produced problems of multicollinearity [70] when both variables were included in the statistical model. Preliminary analysis indicated that maximum daily temperature was a better predictor of mosquito density than minimum daily temperature and so only the latter was included in the final model.

The strength and statistical significance of the association between the meteorological data (daily rainfall and maximum temperature) and Anopheles density is shown by the results of an analysis of deviance given in Table 3. These results indicate that daily rainfall and maximum temperature are strongly and statistically significantly associated with the density of A. gambiae and A. funestus.

The structure of the association between the meteorological covariates and mosquito density over the 7-week lag is depicted in Figure 4. A statistically significant association is suggested at lags (days in the past) where the confidence interval for the multiplicative effect on mean mosquito density does not include 1 . Bearing this in mind, rainfall between 4 and 47 days lag is positively (an increase in rainfall leads to an increase in mosquito density) and statistically significantly associated with the density of A. gambiae (4A). For the density of A. funestus, the association is also positive and statistically significant between 4 and 27 days lag (4C). Maximum 

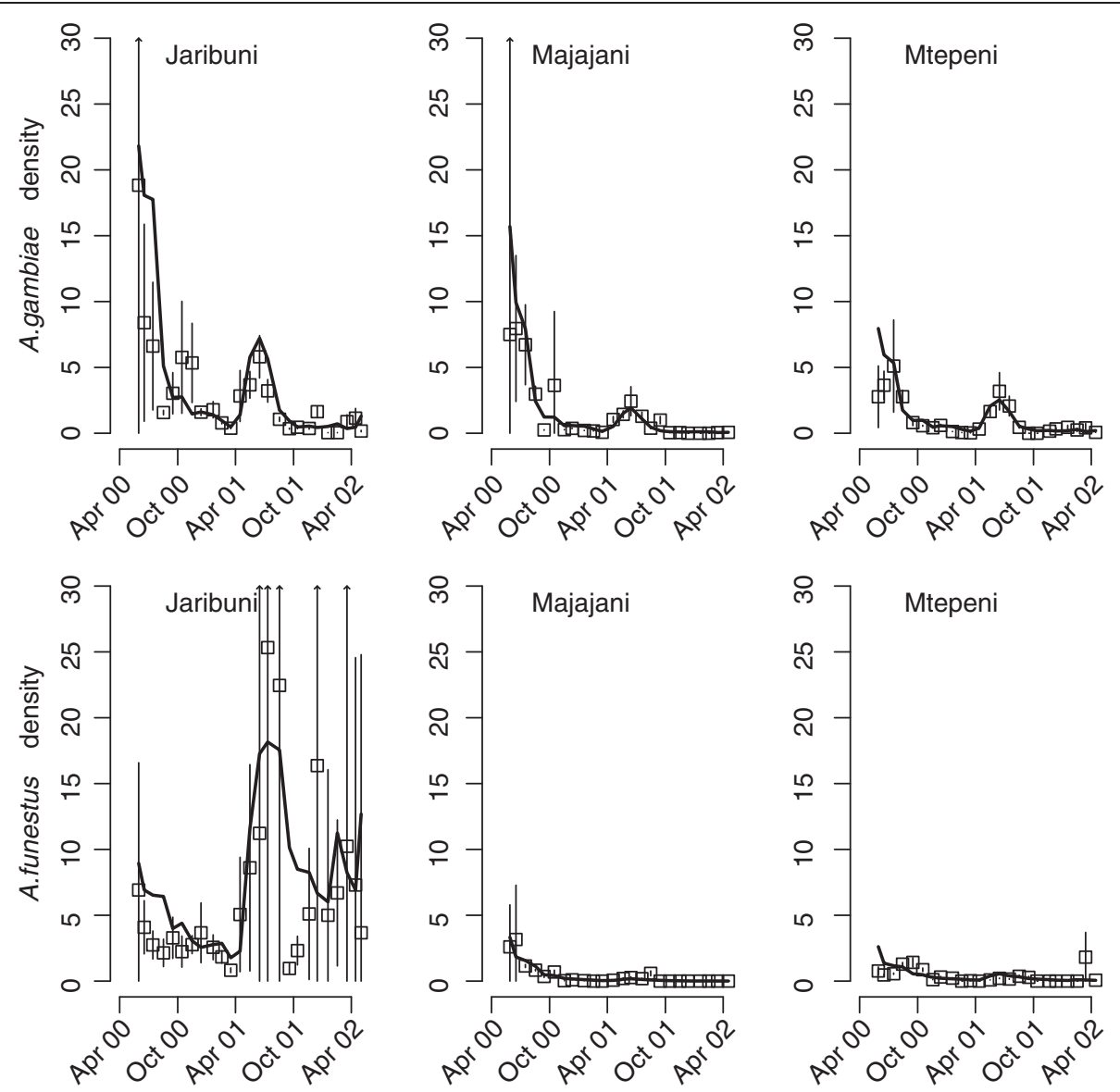

Figure 2 Observed and model-predicted household densities of Anopheles mosquitoes. Depicted in each panel are the observed and model-predicted mosquito densities (the number of mosquitoes per household) in the different sampled sites within Kilifi district; the villages of Jaribuni, Majajani and Mtepeni. Squares represent the observed data averaged (arithmetic means) by month, and the vertical lines indicate the respective $95 \%$ confidence intervals. The solid black lines indicate the mosquito densities predicted from the fitted statistical model. Note that the statistical model was fitted separately to species-specific mosquito counts.

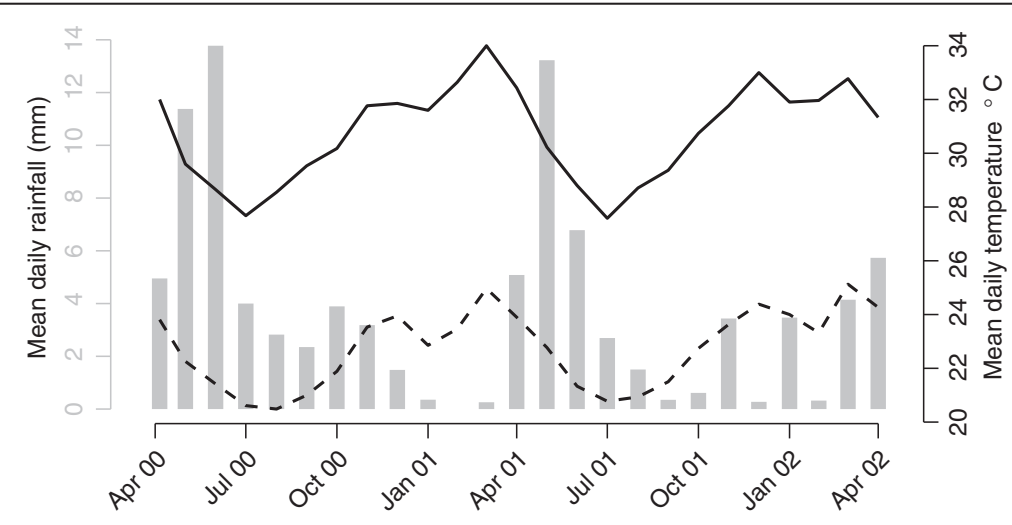

Figure 3 Mean daily rainfall and temperature between April 2000 through April 2002 in Kilifi district, Kenya. The x-axis indicates time from 7 weeks before the start of the study period through to the end in accordance with the maximum and minimum lag periods considered in the statistical model (see main text). The grey bars indicate the mean daily rainfall whose scale is given by the grey $y$-axis on the left hand side. The black solid and dashed lines indicate, respectively, the maximum and minimum mean daily temperatures whose scale is given by the black $y$-axis on the right hand side. 
Table 3 Analysis of deviance of covariates associated with the household density of Anopheles mosquitoes

\begin{tabular}{|c|c|c|c|c|}
\hline Covariate & $\mathrm{DF}^{\mathrm{a}}$ & Species $^{\mathrm{b}}$ & $\Delta \operatorname{Dev}^{c, d}$ & $P$-value \\
\hline \multirow[t]{2}{*}{ Rainfall $^{f}$} & 5 & A. gambiae & 80 & $<0.001$ \\
\hline & & A. funestus & 24 & $<0.001$ \\
\hline \multirow[t]{2}{*}{ Maximum temperature ${ }^{f}$} & 5 & A. gambiae & 57 & $<0.001$ \\
\hline & & A. funestus & 27 & $<0.001$ \\
\hline \multirow{2}{*}{$\begin{array}{l}\text { Days since study start } \\
\text { date } \times \text { village }\end{array}$} & 5 & A. gambiae & 120 & $<0.001$ \\
\hline & & A. funestus & 180 & $<0.001$ \\
\hline \multirow{2}{*}{$\begin{array}{l}\text { Household occupancy the night } \\
\text { before mosquito collection }\end{array}$} & 5 & A. gambiae & 8.9 & 0.11 \\
\hline & & A. funestus & 31 & $<0.001$ \\
\hline \multirow{2}{*}{$\begin{array}{l}\text { Distance of household from } \\
\text { nearest mosquito larval habitat }\end{array}$} & 6 & A. gambiae & 5.2 & 0.51 \\
\hline & & A. funestus & 12 & 0.070 \\
\hline
\end{tabular}

${ }^{\mathrm{a}}$ Degrees of freedom.

${ }^{\mathrm{b}}$ Models fitted separately to species-specific counts per household.

'Difference between the (residual) deviance of the model with all covariates and the model without the covariate in question [46].

${ }^{\mathrm{d}}$ Rounded to 2 significant figures.

e Calculated assuming that, under the null hypothesis (of no statistically significant association between the covariate and the numbers of mosquitoes per household), $\Delta \mathrm{Dev}$ is $X^{2}$ distributed with the indicated degrees of freedom [71] Included as a 4th order polynomial distributed lag term yielding 5 degrees of freedom.

temperature is negatively and statistically significantly associated with the density of $A$. gambiae between 5 and 24 days lag (4B), and with $A$. funestus density between 17 and 35 days lag (4D). A statistically significant positive association between maximum temperature and $A$. gambiae density occurs between 29 and 47 days in the past, and marginally between 45 and 48 days for the density of $A$. funestus.

\section{Household covariates}

The results in Table 3 indicate that the number of people sleeping in the household during the night prior to sampling-hereafter referred to as household occupancy-is strongly and statistically significantly associated with the density of $A$. funestus but not significantly associated with the density of A. gambiae. Figure 5 shows that the density of $A$ funestus increases sharply from household occupancies of 1 to 2 , thereafter remaining relatively constant. The relationship between household occupancy and the density of $A$ gambiae is more linear (and positive), albeit not statistically significant (Table 3 and Figure 5A).

Overall, the distance of the household from the nearest mosquito larval habitat is not a statistically significantly predictor of the density of either Anopheles species (Table 3 and Figure 6). However, the density of $A$. funestus in households situated over 600 metres from the nearest larval habitat is statistically significantly lower $(P$-value $=0.0032)$ than that in households located in the baseline distance category of 101-200 metres for the nearest larval habitat (Figure 6B).
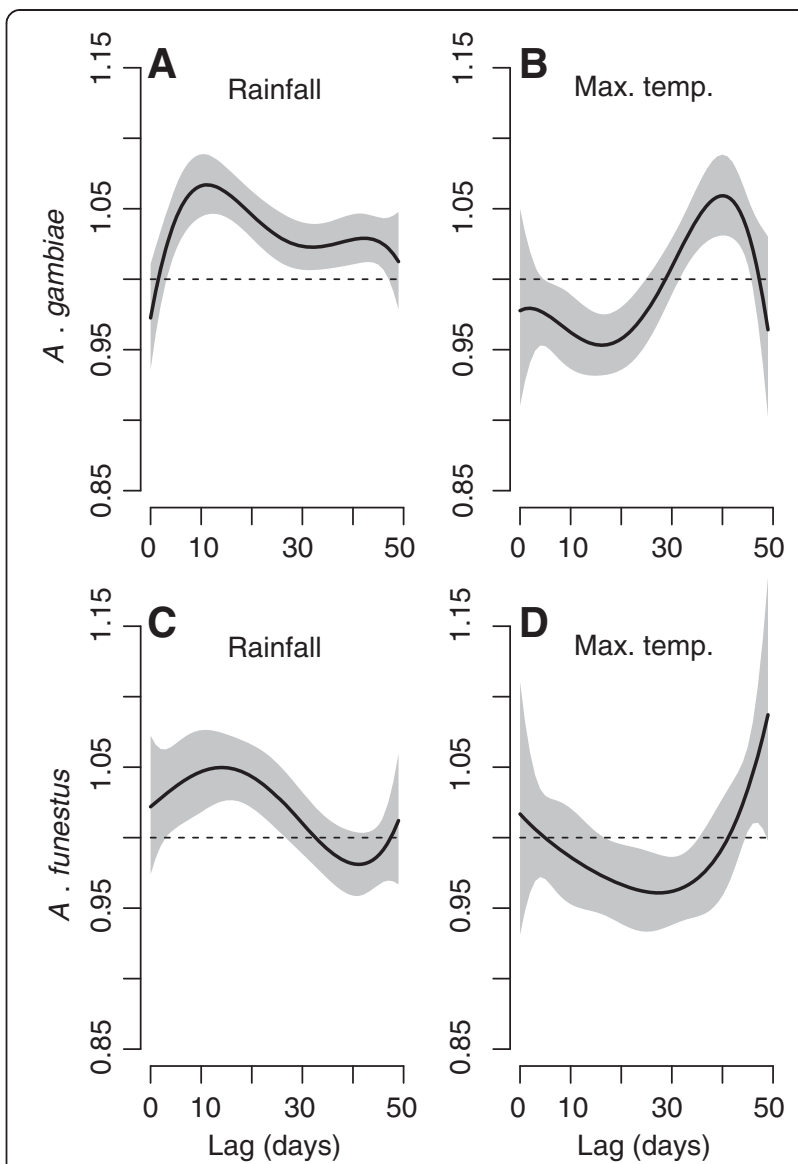

Figure 4 Distributed lag structure of the association between daily rainfall and maximum temperature on the density of Anopheles mosquitoes. The solid lines in each panel represent the model-predicted multiplicative effect on the mean number of mosquitoes per household (mosquito density) associated with either a $1 \mathrm{~mm}$ increase in daily rainfall (Panels $\mathbf{A}$ and $\mathbf{C}$ for $A$. gambiae and $A$. funestus respectively), or a $1^{\circ} \mathrm{C}$ increase in daily temperature at each lag time (Panels $\mathbf{B}$ and $\mathbf{D}$ for $A$. gambiae and A. funestus respectively). The grey shaded areas indicate $95 \%$ confidence intervals. The structure of the relationship between the coefficient and the lag time is constrained by a 4th order polynomial.

\section{Household clustering and overdispersion}

The total amount of extra-Poisson variation (overdispersion) in the observed numbers of mosquitoes per household is quantified by the sum of the variance terms, $\sigma_{\mathrm{H}}^{2}$ and $\sigma_{\mathrm{O}}^{2}$. Parameter $\sigma_{\mathrm{H}}^{2}$ quantifies the amount of overdispersion induced by the clustering of mosquitoes within households. Parameter $\sigma_{\mathrm{O}}^{2}$ quantifies the residual overdispersion having accounted for that induced by household clustering. Thus, from the estimates of $\sigma_{\mathrm{H}}^{2}$ and $\sigma_{\mathrm{O}}^{2}$ given in Table 4 , it can be seen that $0.55 \%[0.4 /(72+$ $0.4)]$ and $0.06 \%[0.22 /(380+0.22)]$ of the total overdispersion in, respectively, A gambiae and A. funestus numbers per household was accounted for by household clustering. Despite this low contribution to the overall 


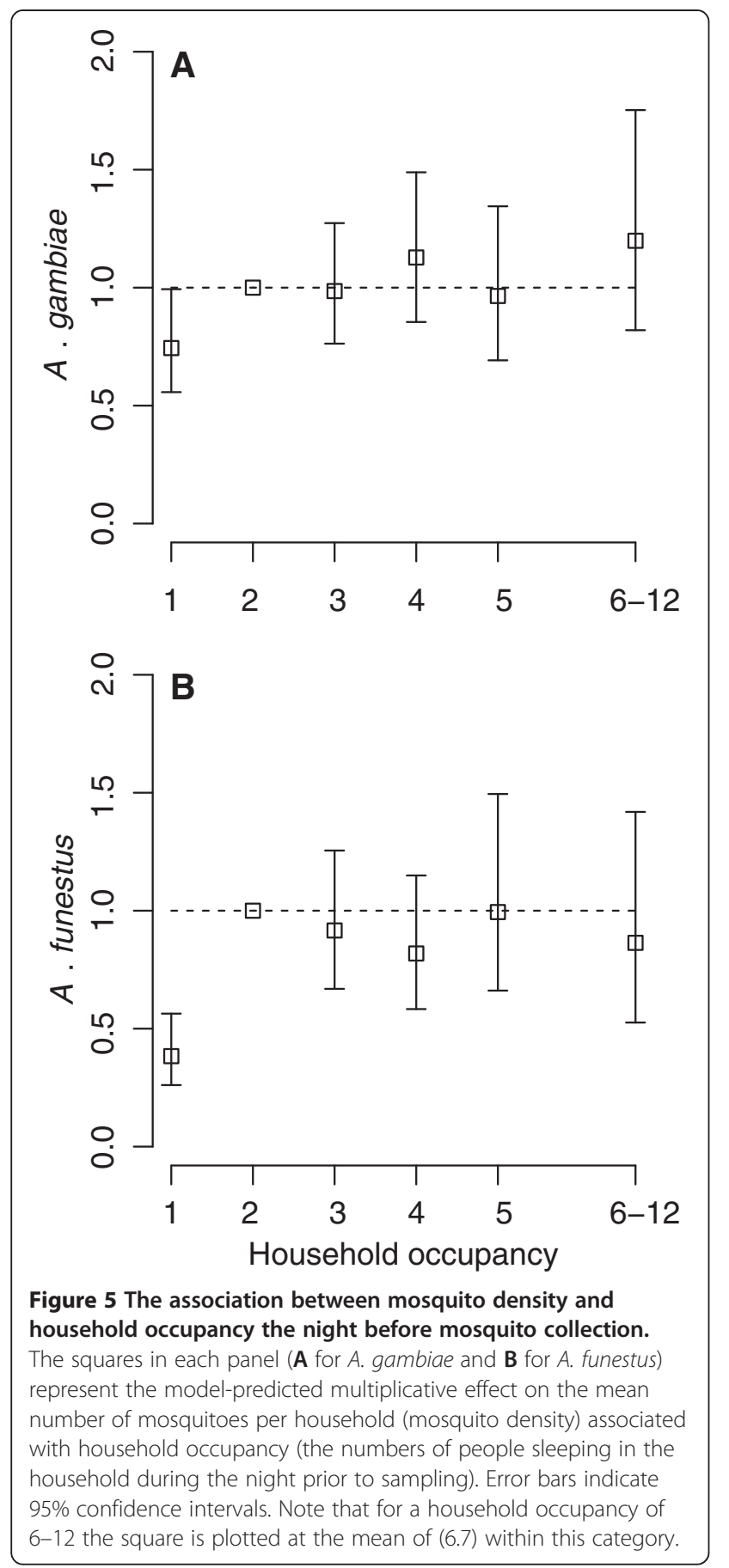

degree of overdispersion, household clustering for both Anopheles species is statistically significant (Table 4).

\section{Discussion}

The mixed PDL models, or PDL GLMMs (Table 2), applied in this analysis have been used to elucidate factors influencing micro-spatial and temporal heterogeneity in the distribution of anopheline vectors of malaria caught within households in Kilifi district, Kenya. Temporal variation in

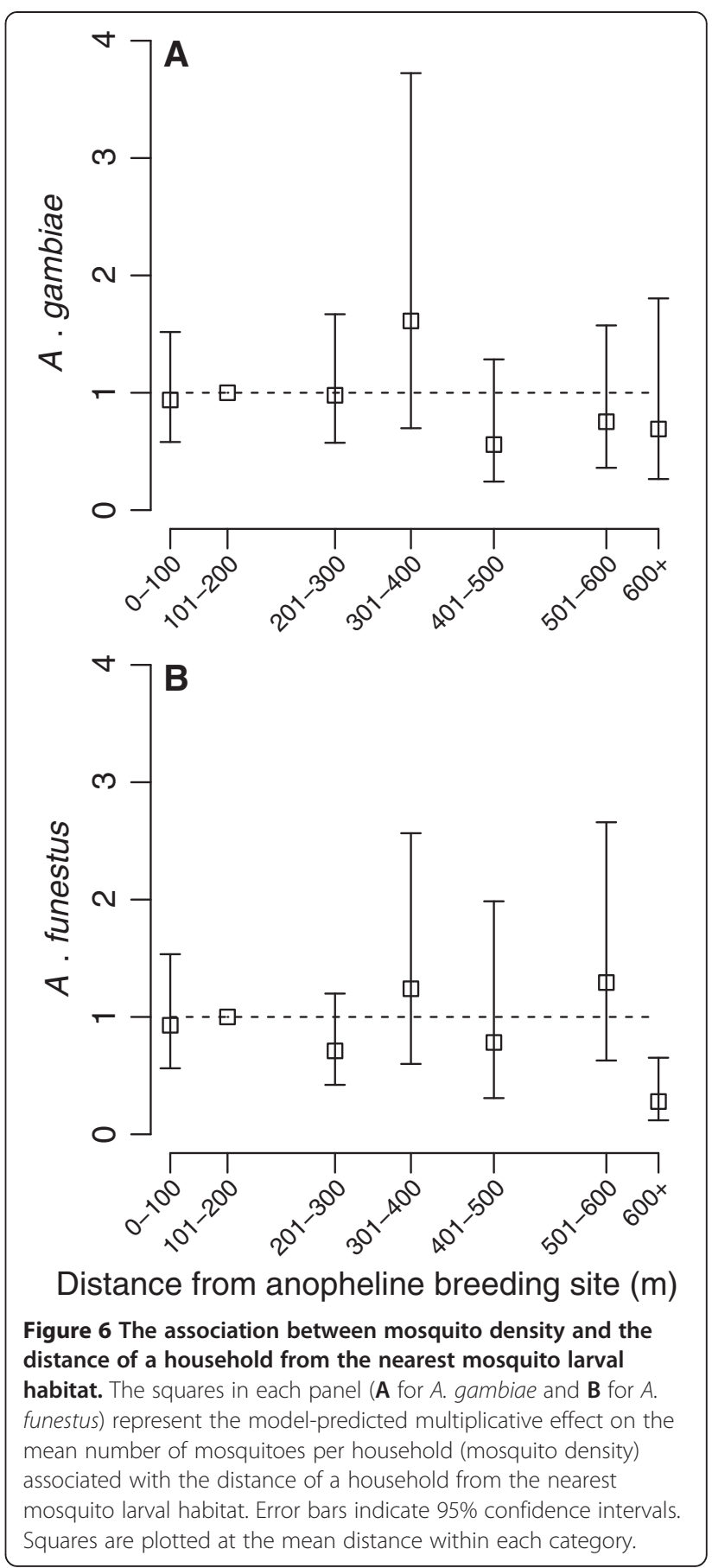

the abundance of $A$. gambiae and A. funestus is driven by seasonal changes in climate, specifically the amount of rainfall from 4 to 47 days in the past (depending on Anopheles species), and maximum daily temperature. The PDL component of the statistical framework has enabled the distributed shape of these relationships to be elucidated for the first time. Micro-spatial variation is related to the distance of households from the nearest anopheline larval habitat, and whether such households have single- or multiple- 
Table 4 Likelihood ratio tests of household clustering and overdispersion in household densities of Anopheles mosquitoes

\begin{tabular}{|c|c|c|c|c|c|}
\hline Parameter & $\mathrm{DF}^{\mathrm{a}}$ & Species & Estimate $^{b}$ & LRS $^{b, c}$ & $P$-value ${ }^{\mathrm{d}}$ \\
\hline \multirow[t]{2}{*}{ Household clustering, $\sigma_{\mathrm{H}}^{2}$} & 1 & A. gambiae & 0.40 & 51 & $<0.001$ \\
\hline & & A. funestus & 0.22 & 18 & $<0.001$ \\
\hline \multirow[t]{2}{*}{ Overdispersion, $\sigma_{\mathrm{O}}^{2}$} & 1 & A. gambiae & 72 & 6300 & $<0.001$ \\
\hline & & A. funestus & 380 & 8100 & $<0.001$ \\
\hline
\end{tabular}

\footnotetext{
${ }^{\mathrm{a}}$ Degrees of freedom.

${ }^{\mathrm{b}}$ Rounded to 2 significant figures.

'Likelihood ratio statistic.

${ }^{d}$ Calculated assuming that, under the null hypothesis (of the parameter in question not statistically significantly improving the log-likelihood of the fitted model), the LRS is $X^{2}$ distributed with the indicated degrees of freedom [71].
}

occupancy by people pernoctating in them on the night prior to sampling. Despite accounting for these explanatory variables, the abundance of mosquitoes is highly overdispersed and clustered among households, effects which were quantified by the GLMM component of the modelling framework.

Strong empirical associations between anopheline abundance and seasonal variations in rainfall and temperature have been demonstrated previously $[5,23,72]$, as have differences in these associations among species [73], although not using a PDL GLMM approach. The PDL models used here permitted a detailed description of the temporally distributed (lagged) nature of these associations which have not been previously elucidated (although the distributed lagged relationship between meteorological variables and cases of clinical malaria have been investigated in a similar fashion [34]). For example, for A. gambiae, rainfall was positively associated with mosquito abundance between 4 and 47 days in the past, with a peak in magnitude at approximately 11 days, and for A. funestus, between 4 and 27 days, with a peak at approximately 14 days. Rainfall acts principally to create and maintain viable larval habitats [74], but the exact shapes of these associations (Figure 4A for A. gambiae, Figure 4C for $A$. funestus) are likely to be the products of a multitude of interacting factors such as the local topology, cumulative effects of rainfall and heterogeneity in mosquito biology, the type, size and shape of larval habitats, in addition to other meteorological variables such as temperature, humidity and evaporation rates.

The distributed association of anopheline abundance with maximum daily temperature is more complex (Figure $4 \mathrm{~B}$ for A. gambiae, Figure 4D for A. funestus); a negative association is indicated at short to medium lags-as observed previously in this area [72] — while a positive association arises at longer lag times. The effect of temperature on mosquito abundance is well documented for the extremes of the species tolerance levels [75,76]. This study sits within these extremes, where the mechanisms by which lagged temperatures may affect mosquito abundance are many and more nuanced, complicating interpretation of statistical (phenomenological) associations. High temperatures may reduce the size and abundance of larval habitats, not only affecting opportunities for breeding, but also altering local ecology, affecting regulatory factors such as the severity of density-dependent competition, the concentration of resources, and water quality among others. Furthermore, temperature influences the development time of anopheline aquatic stages [38-40], possibly interacting with the length of lags for other meteorological variables, such as rainfall. Temperature may also affect adult survival $[77,78]$ and behaviour [79], the latter potentially altering the chance of an individual mosquito being trapped.

Among the micro-spatial determinants of household anopheline abundance, distance to the nearest suitable larval habitat was not, overall, statistically significantly associated with either species (although the density of $A$. funestus was statistically significantly lower for households located over $600 \mathrm{~m}$ from a larval habitat compared with households situated closer to larval habits, see Figure 6). Previous studies have shown that distance to larval habitat is negatively associated with anopheline density [80,81]. The lack of association in this analysis probably reflects, at least in part, a limitation of the GPS data, which were only recorded to an accuracy of approximately 31 metres. A further limitation is that the statistical framework does not include explicit spatial structure. That is, the distribution of larval habitats around individual households could not be considered. This led to the implicit modelling assumption that the dominant source of anophelines was the larval habitat nearest to a household. In principle, the PDL GLMM framework could be incorporated into spatial statistical models. This would permit more powerful interrogation of geo-referenced data and improve the capacity of existing spatial frameworks to capture accurately the effects of meteorological variables.

The intensity of human-derived sensory cues, such as carbon dioxide [82], probably underlies the association between the number of household inhabitants the night prior to mosquito sampling (household occupancy) and the number of anophelines caught; more inhabitants generate more intense cues, attracting more mosquitoes. However, 
the observed strength of this association may be diluted somewhat because houses inhabited by more people tended to be larger (although most houses comprise 2-3 rooms only), catches were made in a single room, and mosquitoes presumably distribute, at least to a certain extent, throughout the house. Moreover, the association may be confounded by the number of occupants in nearby households, which serve to make clusters of high-occupancy households more attractive than low-occupancy household clusters [31]. These factors may explain the lack of a statistically significant association with A. gambiae, a result which is surprising in the context of the highly anthropophilic, anthropophagic and endophagous nature of this species. A lack of association has been observed between the number of inhabitants in a house and malaria prevalence in the region [83].

The species-specific heterogeneity in abundance among the villages is consistent with previous studies in this area [72]. In particular, the markedly greater abundance of $A$. funestus in Jaribuni is attributable to local ecological heterogeneities [23]. Jaribuni lies next to a permanent river (Figure 1B), which provides plentiful and persistent larval habitats. Such habitats are more favourable to A. funestus which tolerate larger, more permanent larval habitats, whereas A. gambiae prefers more localised and transient fresh water habitats $[2,84]$.

Identifying factors driving micro-spatial and temporal heterogeneity in the distribution of anopheline mosquitoes facilitates understanding of the causal pathway which drives the coupled dynamics of malaria transmission. In this regard, this study would have benefited from collecting additional public-health oriented outcome data. For example, measurement of standard entomological indices such as the entomological inoculation rate (EIR) during the study period would have improved our understanding of how the results relate to the incidence of falciparum malaria in the region. Nevertheless, in the context of malaria vector control, understanding spatial and temporal determinants of anopheline abundance can assist with judicious identification of high-risk communities $[8,85]$, or even households, improving the cost-effectiveness of targeted interventions $[81,86]$. Moreover, the lagged nature of meteorological predictors of anopheline abundance can be exploited to predict when malaria outbreaks are likely to occur [32], guiding pre-emptive interventions.

Malaria epidemiology in sub-Saharan Africa has undergone significant changes in recent years [87]. Kilifi, like many areas, has seen a large increase in the distribution of insecticide-treated bednets (ITNs) since these entomological surveys were undertaken $[88,89]$. In other areas changes in bednet use has been associated with changes in entomological ecology, such as declines in A. gambiae s.s. [90]. The influence of the changing intervention landscape on A. funestus is less well understood. This study therefore provides an important baseline assessment of the entomological situation prior to scaling up of ITN distribution and provides a valuable comparative source for ongoing studies in the region.

\section{Conclusions}

The statistical framework applied in this analysis, created by bringing together PDL models and GLMMs, represents a powerful tool for understanding factors underpinning the temporal and spatial distribution of anopheline mosquitoes, vectors of falciparum malaria in the study area. The PDL component of the framework permits modelling of past (lagged) seasonal changes in climate, which affects current mosquito density by mediating the abundance of aquatic larval habitats on a large, macro-spatial scale. The GLMM component permits simultaneous incorporation of finer, micro-spatial determinants of mosquito density while handling robustly any clustering of mosquito counts within units of observation (here, households) and residual overdispersion (extra-Poisson variation).

Continued development and application of appropriate and powerful statistical methods to understand better the determinants of anopheline abundance and malaria incidence will facilitate refinement of public health interventions. Detailed understanding of the impact of spatially and temporally heterogeneous explanatory variables will help to identify and target communities most at risk of malaria, improving the efficiency and cost-effectiveness of control efforts. In particular, a better understanding of the lagged effect of seasonal changes in climate on vector abundance will improve temporal targeting of vector control and other interventions. In turn, including this type of information into early warning systems may assist in the deployment of preventative anti-vectorial measures for malaria control, facilitating better allocation of resources in often resource-poor settings.

\section{Abbreviations}

DF: Degrees of freedom; EIR: Entomological inoculation rate; GLMM: Generalized linear mixed model; GPS: Global positioning system; ITN: Insecticide-treated net; KEMRI: Kenya Medical Research Institute; PDL: Polynomial distributed lag; LRS: Likelihood ratio statistic; PSC: Pyrethrum spray cath; s.I.: Sensu lato; s.s.: Sensu stricto.

\section{Competing interests}

The authors declare that they have no competing interests.

\section{Authors' contributions}

JTM and JMM coordinated the field work, data collection and data curation. JCB and CM provided overall project supervision and scientific guidance. MW, PW and MGB conceived the analytical approach. PW performed preliminary statistical analysis of the data. MW performed the final analysis. MW, PW, MGB and JTM wrote the manuscript. All authors read and approved the final, submitted version.

\section{Acknowledgements}

We thank Punam Amratia, Thomas Churcher and Déirdre Hollingsworth for valuable discussions during earlier stages of the work. Malorie Perry conducted useful literature searches on the relationships between climatic variables and mosquito biology. 


\section{Funding statement}

MW, MGB and JTM acknowledge funding from the Wellcome Trust; PW is supported by the Biology and Biotechnology Scientific Research Council (BBSRC) Training Grant, No. BB/I015957/1; JCB is supported by the National Institutes of Health under Award Numbers R01A1100968 and R01GM093345; MGB and JTM thank the European Commission FP7 Collaborative project TransMalariaBloc (HEALTH-F3-2008-223736). The funders had no role in study design, data collection and analysis, decision to publish, or preparation of the manuscript.

\section{Author details}

'Department of Infectious Disease Epidemiology, School of Public Health, Faculty of Medicine (St Mary's campus), Imperial College London, Norfolk Place, London W2 1PG, UK. ${ }^{2}$ MRC Centre for Outbreak Analysis \& Modelling, Department of Infectious Disease Epidemiology, Imperial College London, London W2 1PG, UK. ${ }^{3}$ Vector Biology Department, KEMRI/Wellcome Trust Research Programme, P.O. Box 230-80108, Kilifi, Kenya. ${ }^{4}$ Department of Public Health Sciences, Miller School of Medicine University of Miami, Miami, USA. ${ }^{5}$ Department of Life Sciences, Division of Cell \& Molecular Biology, Imperial College, South Kensington Campus, London SW7 2AZ, UK.

Received: 18 June 2013 Accepted: 4 October 2013

Published: 28 October 2013

\section{References}

1. Baily NTJ: The Biomathematics of Malaria. Bath: Pitman Press; 1982.

2. Service MW: Mosquito Ecology. Field Sampling Methods. 2nd edition. London: Chapman \& Hall; 1993.

3. Texier G, Machault V, Barragti M, Boutin JP, Rogier C: Environmental determinant of malaria cases among travellers. Malar J 2013, 12:87.

4. Githeko AK, Lindsay SW, Confalonieri UE, Patz JA: Climate change and vector-borne diseases: a regional analysis. Bull World Health Organ 2000, 78:1136-1147.

5. Gage KL, Burkot TR, Eisen RJ, Hayes EB: Climate and vectorborne diseases. Am J Prev Med 2008, 35:436-450

6. Craig MH, Snow RW, le Sueur D: A climate-based distribution model of malaria transmission in sub-Saharan Africa. Parasitol Today 1999, 15:105-111.

7. Ernst KC, Adoka SO, Kowuor DO, Wilson ML, John CC: Malaria hotspot areas in a highland Kenya site are consistent in epidemic and non-epidemic years and are associated with ecological factors. Malar J 2006, 5:78.

8. Kulkarni MA, Desrochers RE, Kerr JT: High resolution niche models of malaria vectors in northern Tanzania: a new capacity to predict malaria risk? PLOS ONE 2010, 5:e9396.

9. Cairns M, Roca-Feltrer A, Garske T, Wilson AL, Diallo D, Milligan PJ, Ghani AC, Greenwood BM: Estimating the potential public health impact of seasonal malaria chemoprevention in African children. Nat Commun 2012, 3:881.

10. Parham PE, Michael E: Modeling the effects of weather and climate change on malaria transmission. Environ Health Perspect 2010, 118:620-626.

11. Geissbühler Y, Kannady K, Chaki PP, Emidi B, Govella NJ, Mayagaya V, Kiama M, Mtasiwa D, Mshinda H, Lindsay SW, Tanner M, Fillinger U, de Castro MC, Killeen GF: Microbial larvicide application by a large-scale, communitybased program reduces malaria infection prevalence in urban Dar es Salaam, Tanzania. PLOS ONE 2009, 4:e5107.

12. Gu W, Utzinger J, Novak RJ: Habitat-based larval interventions: a new perspective for malaria control. Am J Trop Med Hyg 2008, 78:2-6.

13. Kiszewski A, Mellinger A, Spielman A, Malaney P, Sachs SE, Sachs J: A global index representing the stability of malaria transmission. Am J Trop Med Hyg 2004, 70:486-498.

14. Githeko AK, Service MW, Mbogo CM, Atieli FK, Juma FO: Origin of blood meals in indoor and outdoor resting malaria vectors in western Kenya. Acta Trop 1994, 58:307-316.

15. Killeen GF, McKenzie FE, Foy BD, Bogh C, Beier JC: The availability of potential hosts as a determinant of feeding behaviours and malaria transmission by African mosquito populations. Trans $R$ Soc Trop Med Hyg 2001, 95:469-476.

16. Midega JT, Smith DL, Olotu A, Mwangangi JM, Nzovu JG, Wambua Nyangweso G, Mbogo CM, Christophides GK, Marsh K, Bejon P: Wind direction and proximity to larval sites determines malaria risk in Kilifi District in Kenya. Nat Commun 2012, 3:674.
17. Takken W, Knols BG: Odor-mediated behaviour of Afrotropical malaria mosquitoes. Ann Rev Entomol 1999, 44:131-157.

18. Costantini C, Li SG, Della Torre A, Sagnon N, Coluzzi M, Taylor CE: Density, survival and dispersal of Anopheles gambiae complex mosquitoes in a west African Sudan savanna village. Med Vet Entomol 1996, 10:203-219.

19. Parham PE, Pople D, Christiansen-Jucht C, Lindsay S, Hinsley W, Michael E: Modeling the role of environmental variables on the population dynamics of the malaria vector Anopheles gambiae sensu stricto. Malar J 2012, 11:271.

20. Hoshen MB, Morse AP: A weather-driven model of malaria transmission. Malar J 2004, 3:32.

21. Ermert $\mathrm{V}$, Fink AH, Jones AE, Morse AP: Development of a new version of the Liverpool Malaria Model. I. Refining the parameter settings and mathematical formulation of basic processes based on a literature review. Malar I 2011, 10:35.

22. Ageep TB, Cox J, Hassan MM, Knols BG, Benedict MQ, Malcolm CA, Babiker A, El Sayed BB: Spatial and temporal distribution of the malaria mosquito Anopheles arabiensis in northern Sudan: influence of environmental factors and implications for vector control. Malar J 2009, 8:123.

23. Mbogo CM, Mwangangi JM, Nzovu J, Gu W, Yan G, Gunter JT, Swalm C, Keating J, Regens JL, Shililu JI, Githure JI, Beier JC: Spatial and temporal heterogeneity of Anopheles mosquitoes and Plasmodium falciparum transmission along the Kenyan coast. Am J Trop Med Hyg 2003, 68:734-742.

24. Ribeiro JM, Seulu F, Abose T, Kidane G, Teklehaimanot A: Temporal and spatial distribution of anopheline mosquitos in an Ethiopian village: implications for malaria control strategies. Bull World Health Organ 1996, 74:299-305.

25. Peterson AT: Shifting suitability for malaria vectors across Africa with warming climates. BMC Infect Dis 2009, 9:59.

26. Sinka ME, Bangs MJ, Manguin $S$, Coetzee $M$, Mbogo CM, Hemingway J, Patil AP, Temperley WH, Gething PW, Kabaria CW, Okara RM, Van Boeckel T, Godfray HC, Harbach RE, Hay SI: The dominant Anopheles vectors of human malaria in Africa, Europe and the Middle East: occurrence data, distribution maps and bionomic précis. Parasit Vectors 2010, 3:117.

27. Kristan M, Abeku TA, Beard J, Okia M, Rapuoda B, Sang J, Cox J: Variations in entomological indices in relation to weather patterns and malaria incidence in East African highlands: implications for epidemic prevention and control. Malar J 2008, 7:231.

28. Tanser FC, Sharp B, le Sueur D: Potential effect of climate change on malaria transmission in Africa. Lancet 2003, 362:1792-1798.

29. Ferguson HM, Dornhaus A, Beeche A, Borgemeister C, Gottlieb M, Mulla MS, Gimnig JE, Fish D, Killeen GF: Ecology: a prerequisite for malaria elimination and eradication. PLoS Med 2010, 7:e1000303.

30. Garske T, Ferguson NM, Ghani AC: Estimating air temperature and its influence on malaria transmission across Africa. PLOS ONE 2013, 8:e56487.

31. Russell TL, Lwetoijera DW, Knols BG, Takken W, Killeen GF, Kelly-Hope LA: Geographic coincidence of increased malaria transmission hazard and vulnerability occurring at the periphery of two Tanzanian villages. Malar J 2013, 12:24.

32. Teklehaimanot HD, Schwartz J, Teklehaimanot A, Lipsitch M: Weather-based prediction of Plasmodium falciparum malaria in epidemic-prone regions of Ethiopia II. Weather-based prediction systems perform comparably to early detection systems in identifying times for interventions. Malar J 2004, 3:44.

33. Jones AE, Morse AP: Application and validation of a seasonal ensemble prediction system using a dynamic malaria model. J Clim 2010, 23:4202-4215

34. Teklehaimanot HD, Lipsitch M, Teklehaimanot A, Schwartz J: Weather-based prediction of Plasmodium falciparum malaria in epidemic-prone regions of Ethiopia I. Patterns of lagged weather effects reflect biological mechanisms. Malar J 2004, 3:41.

35. Patz JA, Strzepek K, Lele S, Hedden M, Greene S, Noden B, Hay SI, Kalkstein L, Beier JC: Predicting key malaria transmission factors, biting and entomological inoculation rates, using modelled soil moisture in Kenya. Trop Med Int Health 1998, 3:818-827.

36. Cottrell G, Kouwaye B, Pierrat C, le Port A, Bouraima A, Fonton N, Hounkonnou MN, Massougbodji A, Corbel V, Garcia A: Modeling the influence of local environmental factors on malaria transmission in Benin and its implications for cohort study. PLOS ONE 2012, 7:e28812. 
37. Yé Y, Hoshen M, Kyobutungi C, Louis VR, Sauerborn R: Local scale prediction of Plasmodium falciparum malaria transmission in an endemic region using temperature and rainfall. Glob Health Action 2009, 2. doi: 10.3402/gha.v2i0.1923.

38. Bayoh MN, Lindsay SW: Effect of temperature on the development of the aquatic stages of Anopheles gambiae sensu stricto (Diptera: Culicidae). Bull Entomol Res 2003, 93:375-381.

39. Kirby MJ, Lindsay SW: Effect of temperature and inter-specific competition on the development and survival of Anopheles gambiae sensu stricto and An. arabiensis larvae. Acta Trop 2009, 109:118-123.

40. Lyons CL, Coetzee M, Chown SL: Stable and fluctuating temperature effects on the development rate and survival of two malaria vectors, Anopheles arabiensis and Anopheles funestus. Parasit Vectors 2013, 6:104.

41. Patz JA, Olson SH: Malaria risk and temperature: influences from global climate change and local land use practices. Proc Natl Acad Sci U S A 2006, 103:5635-5636.

42. Galardo AKR, Zimmerman RH, Lounibos LP, Young LJ, Galardo CD, Arruda M, Couto AARD: Seasonal abundance of anopheline mosquitoes and their association with rainfall and malaria along the Matapi River, Amapi, Brazil. Med Vet Entomol 2009, 23:335-349.

43. Alemu A, Abebe G, Tsegaye W, Golassa L: Climatic variables and malaria transmission dynamics in Jimma town. South West Ethiopia. Parasit Vectors 2011, 4:30

44. Zacarias OP, Andersson M: Spatial and temporal patterns of malaria incidence in Mozambique. Malar J 2011, 10:189.

45. Diggle P, Heagerty P, Liang K-Y, Zeger S: Analysis of Longitudinal Data. Oxford: Oxford University Press; 2002.

46. McCullagh P, Nelder JA: Generalized Linear Models. 2nd edition. London: Chapman \& Hall; 1989

47. Paterson S, Lello J: Mixed models: getting the best use of parasitological data. Trends Parasitol 2003, 19:370-375.

48. Bolker BM, Brooks ME, Clark CJ, Geange SW, Poulsen JR, Stevens MH, White JS Generalized linear mixed models: a practical guide for ecology and evolution. Trends Ecol Evol 2009, 24:127-135.

49. Mosha FW, Mutero CM: Separation of Anopheles merus from freshwater Anopheles gambiae by salinity tolerance test and morphological characters. Parassitologia 1982, 24:255-264.

50. Mosha FW, Petrarca V: Ecological studies on Anopheles gambiae complex sibling species on the Kenya coast. Trans R Soc Trop Med Hyg 1983, 77:344-345.

51. Mbogo CN, Baya NM, Ofulla AV, Githure Jl, Snow RW: The impact of permethrin-impregnated bednets on malaria vectors of the Kenyan coast. Med Vet Entomol 1996, 10:251-259.

52. Mbogo CN, Glass GE, Forster D, Kabiru EW, Githure Jl, Ouma JH, Beier JC: Evaluation of light traps for sampling anopheline mosquitoes in Kilifi, Kenya. J Am Mosa Control Assoc 1993, 9:260-263.

53. Mbogo CN, Kabiru EW, Muiruri SK, Nzovu JM, Ouma JH, Githure Jl, Beier JC: Bloodfeeding behavior of Anopheles gambiae s.l. and Anopheles funestus in Kilifi District, Kenya. J Am Mosa Control Assoc 1993, 9:225-227.

54. Mbogo CNM, Snow RW, Khamala CPM, Kabiru EW, Ouma JH, Githure Jl, Marsh K, Beier JC: Relationships between Plasmodium falciparum transmission by vector populations and the incidence of severe disease at 9 sites on the Kenyan coast. Am J Trop Med Hyg 1995, 52:201-206.

55. Mwangangi JM, Mbogo CM, Nzovu JG, Kabiru EW, Mwambi H, Githure JI, Beier JC: Relationships between body size of Anopheles mosquitoes and Plasmodium falciparum sporozoite rates along the Kenya coast. J Am Mosa Control Assoc 2004, 20:390-394.

56. Bejon P, Williams TN, Liljander A, Noor AM, Wambua J, Ogada E, Olotu A Osier FH, Hay SI, Färnert A, Marsh K: Stable and unstable malaria hotspots in longitudinal cohort studies in Kenya. PLoS Med 2010, 7:e1000304.

57. Okiro EA, Alegana VA, Noor AM, Snow RW: Changing malaria intervention coverage, transmission and hospitalization in Kenya. Malar J 2010, 9:285.

58. O'Meara WP, Bejon P, Mwangi TW, Okiro EA, Peshu N, Snow RW, Newton CR, Marsh K: Effect of a fall in malaria transmission on morbidity and mortality in Kilifi, Kenya. Lancet 2008, 372:1555-1562.

59. Mwangangi JM, Mbogo CM, Orindi BO, Muturi EJ, Midega JT, Nzovu J, Gatakaa H, Githure J, Borgemeister C, Keating J, Beier JC: Shifts in malaria vector species composition and transmission dynamics along the Kenyan coast over the past 20 years. Malar J 2013, 12:13.

60. World Health Organization: Manual on Practical Entomology in Malaria. Part 2: Methods and Techniques. Geneva: World Health Organization; 1975.
61. Gillies MT, Coetzee M: Supplement to the Anophelinae of Africa South of the Sahara (Afrotropical Region). Johannesburg: South African Institute of Medical Research; 1987

62. Scott JA, Brogdon WG, Collins FH: Identification of single specimens of the Anopheles gambiae complex by the polymerase chain reaction. Am J Trop Med Hyg 1993, 49:520-529.

63. Koekemoer LL, Kamau L, Hunt RH, Coetzee M: A cocktail polymerase chain reaction assay to identify members of the Anopheles funestus (Diptera: Culicidae) group. Am J Trop Med Hyg 2002, 66:804-811.

64. R Development Core Team, R Development Core Team: R: A Language and Environment for Statistical Computing. Vienna: R Foundation for Statistical Computing; 2011.

65. Elston DA, Moss R, Boulinier T, Arrowsmith C, Lambin X: Analysis of aggregation, a worked example: numbers of ticks on red grouse chicks. Parasitology 2001, 122:563-569.

66. Bolker BM: Ecological Models and Data in R. Prinecton: Princeton University Press; 2008.

67. Almon S: The distributed lag between capital appropriations and net expenditure. Econometrica 1965, 33:178-196.

68. le Sueur D, Sharp BL: Temperature-dependent variation in Anopheles merus larval head capsule width and adult wing length: implications for anopheline taxonomy. Med Vet Entomol 1991, 5:55-62.

69. Rueda LM, Patel KJ, Axtell RC, Stinner RE: Temperature-dependent development and survival rates of Culex quinquefasciatus and Aedes aegypti (Diptera: Culicidae). J Med Entomol 1990, 27:892-898.

70. Draper NR, Smith H: Applied Regression Analysis. New York: John Wiley \& Sons; 1998.

71. Kirkwood BR, Sterne JAC: Essential Medical Statistics. 2nd edition. Oxford: Blackwell Science; 2003.

72. Kelly-Hope LA, Hemingway J, McKenzie FE: Environmental factors associated with the malaria vectors Anopheles gambiae and Anopheles funestus in Kenya. Malar J 2009, 8:268.

73. Lyons CL, Coetzee M, Terblanche JS, Chown SL: Thermal limits of wild and laboratory strains of two African malaria vector species, Anopheles arabiensis and Anopheles funestus. Malar J 2012, 11:226.

74. Koenraadt CJ, Githeko AK, Takken W: The effects of rainfall and evapotranspiration on the temporal dynamics of Anopheles gambiae s.s. and Anopheles arabiensis in a Kenyan village. Acta Trop 2004, 90:141-153.

75. Sinka ME, Bangs MJ, Manguin S, Chareonviriyaphap T, Patil AP, Temperley WH, Gething PW, Elyazar IR, Kabaria CW, Harbach RE, Hay SI: The dominant Anopheles vectors of human malaria in the Asia-Pacific region: occurrence data, distribution maps and bionomic précis. Parasit Vectors 2011, 4:89.

76. Lindsay SW, Parson L, Thomas CJ: Mapping the ranges and relative abundance of the two principal African malaria vectors, Anopheles gambiae sensu stricto and An. arabiensis, using climate data. Proc Biol Sci 1998, 265:847-854.

77. Okech BA, Gouagna LC, Killeen GF, Knols BG, Kabiru EW, Beier JC, Yan G, Githure JI: Influence of sugar availability and indoor microclimate on survival of Anopheles gambiae (Diptera: Culicidae) under semifield conditions in western Kenya. J Med Entomol 2003, 40:657-663.

78. Kirby MJ, Lindsay SW: Responses of adult mosquitoes of two sibling species, Anopheles arabiensis and A. gambiae s.s. (Diptera: Culicidae), to high temperatures. Bull Entomol Res 2004, 94:441-448.

79. Platt RB, Collins CL, Witherspoon JP: Reactions of Anopheles quadrimaculatus Say to moisture, temperature and light. Ecol Monogr 1957, 27:303-324.

80. Amek N, Bayoh N, Hamel M, Lindblade KA, Gimnig JE, Odhiambo F, Laserson KF, Slutsker L, Smith T, Vounatsou P: Spatial and temporal dynamics of malaria transmission in rural Western Kenya. Parasit Vectors 2012, 5:86

81. Zhou G, Munga S, Minakawa N, Githeko AK, Yan G: Spatial relationship between adult malaria vector abundance and environmental factors in western Kenya highlands. Am J Trop Med Hyg 2007, 77:29-35.

82. Gillies MT: The role of carbon-dioxide in host-finding by mosquitos (Diptera, Culicidae) - a review. Bull Entomol Res 1980, 70:525-532.

83. Winskill P, Rowland M, Mtove G, Malima RC, Kirby MJ: Malaria risk factors in north-east Tanzania. Malar J 2011, 10:98.

84. Gimnig JE, Ombok M, Kamau L, Hawley WA: Characteristics of larval anopheline (Diptera: Culicidae) habitats in Western Kenya. J Med Entomol 2001, 38:282-288. 
85. Mboera LE, Senkoro KP, Mayala BK, Rumisha SF, Rwegoshora RT, Mlozi MR, Shayo EH: Spatio-temporal variation in malaria transmission intensity in five agro-ecosystems in Mvomero district, Tanzania. Geospat Health 2010, 4:167-178.

86. Carter R, Mendis KN, Roberts D: Spatial targeting of interventions against malaria. Bull World Health Organ 2000, 78:1401-1411.

87. O'Meara WP, Mangeni JN, Steketee R, Greenwood B: Changes in the burden of malaria in sub-Saharan Africa. Lancet Infect Dis 2010, 10:545-555.

88. Noor AM, Amin AA, Akhwale WS, Snow RW: Increasing coverage and decreasing inequity in insecticide-treated bed net use among rural Kenyan children. PLoS Med 2007, 4:e255.

89. Noor AM, Mutheu JJ, Tatem AJ, Hay SI, Snow RW: Insecticide-treated net coverage in Africa: mapping progress in 2000-07. Lancet 2009, 373:58-67.

90. Lindblade KA, Gimnig JE, Kamau L, Hawley WA, Odhiambo F, Olang G, Ter Kuile FO, Vulule JM, Slutsker L: Impact of sustained use of insecticidetreated bednets on malaria vector species distribution and culicine mosquitoes. J Med Entomol 2006, 43:428-432.

doi:10.1186/1756-3305-6-311

Cite this article as: Walker et al:: Temporal and micro-spatial

heterogeneity in the distribution of Anopheles vectors of malaria along

the Kenyan coast. Parasites \& Vectors 2013 6:311.

\section{Submit your next manuscript to BioMed Central and take full advantage of:}

- Convenient online submission

- Thorough peer review

- No space constraints or color figure charges

- Immediate publication on acceptance

- Inclusion in PubMed, CAS, Scopus and Google Scholar

- Research which is freely available for redistribution 